\title{
Hydrodynamics of surface swimming in leopard frogs (Rana pipiens)
}

\section{Citation}

Johansson, L. C. 2004. Hydrodynamics of Surface Swimming in Leopard Frogs (Rana Pipiens). Journal of Experimental Biology 207, no. 22: 3945-3958. doi:10.1242/jeb.01258.

\section{Published Version}

doi:10.1242/jeb.01258

\section{Permanent link}

http://nrs.harvard.edu/urn-3:HUL.InstRepos:30510314

\section{Terms of Use}

This article was downloaded from Harvard University's DASH repository, and is made available under the terms and conditions applicable to Other Posted Material, as set forth at http:// nrs.harvard.edu/urn-3:HUL.InstRepos:dash.current.terms-of-use\#LAA

\section{Share Your Story}

The Harvard community has made this article openly available.

Please share how this access benefits you. Submit a story.

Accessibility 
doi: $10.1242 /$ jeb. 01258

\title{
Hydrodynamics of surface swimming in leopard frogs (Rana pipiens)
}

\author{
L. Christoffer Johansson ${ }^{1, *}$ and George V. Lauder ${ }^{2}$ \\ ${ }^{1}$ Dept of Theoretical Ecology, Lund University, Ecology Building, SE-223 62 Lund, Sweden and ${ }^{2}$ Dept of Organismic \\ and Evolutionary Biology, Harvard University, 26 Oxford Street, Cambridge, MA 02138, USA \\ *Author for correspondence (e-mail: christoffer.johansson@teorekol.lu.se)
}

Accepted 23 August 2004

\section{Summary}

The kinematics of swimming frogs have been studied extensively in the past and, based on these results, hypotheses regarding the hydrodynamics of frog swimming can be generated. To test these hypotheses we used digital particle image velocimetry (DPIV) to quantify the flow structure of the wake produced by the feet during the propulsion phase of the kick of surface swimming frogs (Rana pipiens). These frogs use two different gaits, asynchronous and synchronous kicking, and the magnitude of the thrust produced by the feet differs between asynchronous $\left(34 \pm 5.4 \mathrm{mN}^{-1}\right.$ foot $\left.^{-1}\right)$ and synchronous kicking $\left(\mathbf{7 1} \pm 13.3 \mathrm{mN}\right.$ foot $\left.^{-1}\right)$, as does maximum swimming speed, with higher swimming speed and forces produced during the synchronous kicks. Previous studies have suggested that an interaction between the feet, resulting in a single posteriorly directed fluid jet, as the feet come together at the end of synchronous kicks, may augment force production. Our results show, however, that each foot produces its own distinct vortex ring, in both asynchronous and synchronous kicking of the feet. There is no evidence of a central jet being produced even during powerful synchronous kicks (maximum thrust calculated was
$264 \mathrm{mN}$ foot $\left.^{-1}\right)$. An alternative mechanism of force production could be the lift-based paddling recently suggested for delta-shaped feet of swimming birds. However, the orientation of the vortex rings generated by the feet is almost perpendicular to the swimming direction for both gaits and there is only a slight asynchrony of the shedding of the distal (start) and proximal (stop) vortex rings, which is different from what would be expected by a dominantly lift-based mechanism. Thus, our results do not support lift as a major mechanism contributing to thrust. Instead, our data support the hypothesis that propulsion is based on drag and acceleration reaction forces where the thrust is generated by separated, but attached, vortex rings on the suction side of the feet, resulting in vortices that are shed behind the frogs during both asynchronous and synchronous kicking.

Supplementary material available online at http://jeb.biologists.org/cgi/content/full/207/22/3945/DC1

Key words: frog, Rana pipiens, anuran, amphibian, DPIV, digital particle image velocimetry, paddling, kicking, swimming, locomotion, kinematics, hydrodynamics, vortex ring, limb, foot.

\section{Introduction}

Jumping and swimming behavior in frogs has been the focus of a number of studies with the goal of understanding both the rapid movements involved in the jump, from the perspective of muscle performance, and the biomechanical changes involved in making a transition from terrestrial to aquatic locomotion (Lutz and Rome, 1994; Marsh, 1994; Marsh and John-Alder, 1994; Peters et al., 1996; Gillis and Biewener, 2000; Gillis and Blob, 2001; Nauwelaerts et al., 2001; Kargo et al., 2002a,b).

Both jumping and swimming are important escape behaviors in frogs, and some studies have suggested the possibility of coadaptation between jumping and swimming such that locomotion in any one environment is a compromise resulting from the requirement that frogs should be able to move effectively in both environments (Emerson and De Jongh, 1980; Nauwelaerts and Aerts, 2002). Some recent work has suggested differences in the kinematic pattern between jumping and swimming in frogs (Nauwelaerts and Aerts, 2003), indicating the possibility of adaptive behaviors associated specifically with swimming. One basis for differences between jumping and swimming performance may be found in the different physical properties of the ground and the water as platforms for force production. While most terrestrial substrates are effectively not deformable and are almost unaffected by forces exerted on them, water yields when pushed upon, resulting in a different resistance to the limb motion. In water, the force produced, and thereby the resistance to the motion of the feet, depends strongly on the actual kinematics of the kicking feet. To get an understanding of the force production during limb motion in water and to understand the effect of previously recorded muscle activation patterns, it is necessary to study the hydrodynamic basis of the force production and to measure 
directly forces produced by swimming animals. In other words, it is necessary to study the flow around the feet and in the wake of the swimming frogs to better understand the mechanisms of force production in the water to provide a basis for comparisons with terrestrial locomotion.

Although swimming frogs may utilize two distinct gaits, asynchronous and synchronous kicking (Calow and Alexander, 1973; Nauwelaerts and Aerts, 2002), most previous research has focused on the more powerful synchronous kicks. This focus has been motivated by the desire to compare swimming and jumping in frogs as both legs move together during a terrestrial jump. However, the use of two gaits suggests the possibility of different hydrodynamic mechanisms being used for asynchronous and synchronous kicks, and one goal of the present study is to evaluate experimentally and compare the hydrodynamics of both types of kicks in leopard frogs swimming in the water. In addition, previous work on aquatic locomotion in frogs has suggested specific hypotheses that are amenable to test using experimental fluid mechanical analyses of frog swimming. Gal and Blake (1988b) suggested that the first part of the propulsive kick is based on drag (fluid resistance to motion) and acceleration reaction (fluid resistance to acceleration) and is followed by a phase when a jet is produced as the feet come together at the end of the power stroke. This hypothesis was proposed because of a mismatch between the forces required to accelerate the frog and the result of a blade-element analysis of the drag and acceleration reaction forces on the feet. An alternative hypothesis, which could explain the observed acceleration pattern, is that the feet function as suggested for the feet of swimming birds (Johansson and Norberg, 2003). In this case, the feet could initially produce thrust using a drag- and acceleration-reactionbased mechanism followed by a lift-based (force generation perpendicular to the motion of the feet) phase. Predictions from this hypothesis include a relatively early shedding of a start vortex and relatively large vortex rings being generated. This mechanism was suggested only recently for birds and has not yet been examined in frogs.

The hydrodynamics of swimming frogs have previously been studied indirectly, using kinematic information to infer the mechanics of the force production (Gal and Blake, 1988a,b). Here, we use digital particle image velocimetry (DPIV), a technique that we have previously applied to study locomotor hydrodynamics in fishes (e.g. Drucker and Lauder, 1999, 2002; Lauder and Drucker, 2002; Tytell and Lauder, 2004), which makes it possible to measure quantitatively the flow around the kicking feet and in the wake of swimming frogs. Our aim is thus to test the hydrodynamic hypotheses outlined above and to describe the wake and vortex generation by surface-swimming leopard frogs using the two gaits.

\section{Materials and methods} Animals

Leopard frogs (Rana pipiens Schreber) were obtained from a commercial supplier (NASCO, Fort Atkinson, WI, USA) and were kept in tanks $(26 \times 62 \mathrm{~cm}$ bottom area) containing Eco earth and sphagnum moss (Zoo Med Laboratories, San Luis Obispo, CA, USA). Each tank had a $22 \times 22 \mathrm{~cm}, 4 \mathrm{~cm}$-deep container filled with water, allowing the frogs to fully submerge themselves. The frogs were fed live crickets supplemented with vitamins. All data were collected within the first month of captivity from five animals with body masses ranging from 30.7 to $42.8 \mathrm{~g}$ (mean $36.1 \mathrm{~g}$ ). Animal procedures were in accordance with approved animal protocols (Harvard University, USA). After the completion of the study, the animals were euthanized with an overdose of MS-222, then weighed and the feet photographed to estimate the surface area. Mean foot area was $4.29 \pm 0.30 \mathrm{~cm}^{2}(N=5)$.

\section{Kinematics}

Three-dimensional kinematics of swimming frogs were quantified by digitizing (using Scion Image; Scioncorp, www.scioncorp.com) morphological marks on the feet of frogs from high-speed video images of frogs swimming in a flow tank. The flow was kept at a constant speed of approximately $0.076 \mathrm{~m} \mathrm{~s}^{-1}$. The filming was conducted using two synchronous high-speed video cameras (Redlake Motionscope PCI 500; Redlake, San Diego, CA, USA) operating at 250 frames s $^{-1}$. The video sequences were taken with one of the cameras filming, though a mirror, the ventral view of the frog while the other camera filmed, synchronously, either the lateral view or, through a mirror placed downstream at $45^{\circ}$ in the flowtank, the caudal view. The points digitized were the tips of all toes, the heels and the ankles. Kinematic data from the two views were then corrected for any parallax error using direct linear transformation (DLT). Owing to a slight shift in the rear-view mirror between the calibration images and the kinematics images, the rear-view calibration image was shifted to compensate for the misalignment. The actual shift was determined by the visual shift between the images as well as by a minimization of the residual error of a number of test points. The median residual error in the data after the DLT was $0.85 \mathrm{~mm}$, which includes digitization error.

In addition to the detailed kinematics of the motion of the feet, the average coordinates of three marks on the body of the frogs and the tip of the third toe, from a more zoomed-out view, were used to calculate swimming velocity and foot velocity from representative sequences. Body and foot velocity were calculated, using Matlab 6.5 (MathWorks Inc., Natick, MA, USA), as the first derivative of a smoothing spline of the time-position curve of the average body coordinate and the tip of the third toe, respectively.

\section{Digital particle image velocimetry (DPIV)}

DPIV is a technique where the motion of small, reflective particles, suspended in a fluid (for example air or water), is estimated by analyzing the changes in the intensity pattern of small sub-samples of, in our case, successive digital images (e.g. Drucker and Lauder, 1999; Lauder 2000; Lauder et al., 2002; Nauen and Lauder, 2002). The result is presented as velocity vectors distributed homogeneously over the analyzed image. 
Based on these vectors, it is then possible to calculate relevant parameters, such as vorticity and circulation. We used hollow, silver-coated, glass beads as reflective particles, as in previous research. To keep the particles suspended and to increase the time the frogs spent within the field of view, we used a flow tank (mean flow velocity of $0.093 \mathrm{~m} \mathrm{~s}^{-1}$ for the analyzed sequences). The particles were illuminated using an $8 \mathrm{~W}$ continuous-wave argon-ion laser (Coherent Inc., Santa Clara, CA, USA) focused into a thin light sheet. Preliminary analyses indicated high flow velocities close to the feet and in the vortex ring jets. Therefore, for all experiments we used a high-resolution $(1280 \times 1024$ pixels at 8-bit gray scale), high-speed video system (NAC Hi-Dcam II, NAC Image Technology, Simi Valley, CA, USA) filming at 500 frames s$^{-1}$ to obtain high-resolution sequences of flow around the feet. Despite the high frame rate, displacement between images was sometimes suboptimal, resulting in underestimates of the force generated. To avoid blurring, the shutter speed was set as fast as the available light allowed $(1 / 1000 \mathrm{~s})$. As a consequence of the high film rate and short shutter speeds, the light available was barely enough for filming, and the camera's electronic gain was turned up. This, however, resulted in noticeable static image noise. The noise was removed before the PIV analysis by subtracting the average image intensity of hundreds of frames. The resulting image was then adjusted to obtain an image intensity where the particles were clearly visible. All image processing was performed in custom programs written in Matlab.

The main source of propulsive force is from the kicking of the hind limbs (Peters et al., 1996), and we therefore concentrated our study on the flow around the feet of the frogs. We chose to study only the propulsive kick of swimming frogs, as this phase has previously been shown to be the determinant of maximum and average swimming speed (Nauwelaerts et al., 2001). Previous studies have also indicated two different modes of locomotion in swimming frogs: asynchronous and synchronous kicking (Calow and Alexander, 1973; Nauwelaerts and Aerts, 2002). We studied both types of locomotion. To obtain an understanding of the threedimensional structure of the flow behind the frogs, we ran two sets of experiments: one with a horizontal light sheet to image the $x z$ plane (filming from the ventral view) and one with a vertical light sheet to image the $x y$ plane (filming from the lateral view); see Fig. 1. To minimize image disturbance, due to reflections of light at the surface, only sequences where the vortex ring did not interact significantly with the surface were used for the vertical light sheet. The same control was not possible for the horizontal light sheet, but the light sheet was positioned well below the surface. Thus, interaction between the feet and the surface was minimized to the extent possible experimentally, although we cannot rule out some possible surface effects on the initial formation of the vortex rings. In addition to the camera used for the DPIV, we used a Redlake camera to simultaneously film a zoomed-out ventral view during DPIV experiments. This camera was directed further upstream to allow us to determine the swimming velocity of the frogs for most of the sequences. For the DPIV sequences
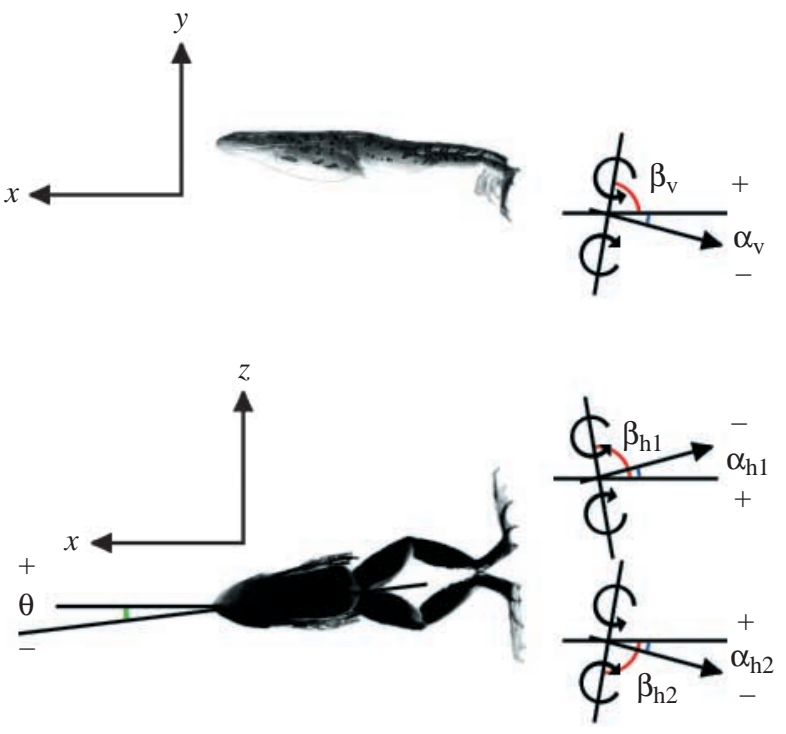

Fig. 1. Definition of $x$-, $y$ - and $z$-axis orientations, jet angle $(\alpha)$, vortex ring angle $(\beta)$ and swimming direction $(\theta)$ in the lateral (top) and ventral (bottom) views, used to measure the vortex wake and body kinematics of swimming leopard frogs. Subscripts $v$ and h represent light sheet orientation during the DPIV experiments, i.e. vertical and horizontal, respectively.

where a ventral view from the Redlake camera was available, we calculated the maximum swimming velocity at the end of the power stroke. The velocity was calculated from the slope of the time-position curve of a well-defined mark on the body over four images, starting when the feet reached their most caudal position relative to the body. To calculate the actual swimming velocity, we then added the flow velocity of the tank.

The PIV analysis was conducted using a code written by E. Tytell for Matlab v.6.5 (Tytell and Lauder, 2004). The analysis was done by cross-correlation of successive images following the procedures of Hart (2000), with an initial search window size of $64 \times 64$ pixels and a final window size of $32 \times 32$ pixels with a $50 \%$ overlap. This resulted in 5120 vectors per image. The vectors were smoothed using an adaptive Gaussian smoothing routine, based on an optimal window size calculated according to Agui and Jimenez (1987). Ten image pairs, from after the completion of the kick, from each sequence were analyzed, resulting in 10 vector fields per sequence. The foot or feet were often still in the image, and sometimes the DPIV analysis picked up the motion of the feet, generating vectors going opposite to what would be expected from the flow. While this proved useful in allowing us to directly calculate the velocities of different parts of the feet during the kick using DPIV, smoothing such images resulted in artefactual vector patterns for nearby water flows. Therefore, the vector field (of the 10 available for each sequence) showing the least amount of disturbances was chosen for further analysis. For the figures shown in this paper, a number of sequences were analyzed in full to illustrate the flow field close to the feet and the development of the flow during the kick. 
To calculate the propulsive force, we determined the size and circulation of the vortex rings produced by the feet. The location and circulation of each vortex center was measured, after subtracting the mean flow vector from the vector field [in Tecplot 9.1 (Tecplot Inc., Bellevue, WA, USA) using custom-written software]. The area over which the vorticity was integrated to estimate the circulation was determined by finding the area that maximized the circulation within a limit
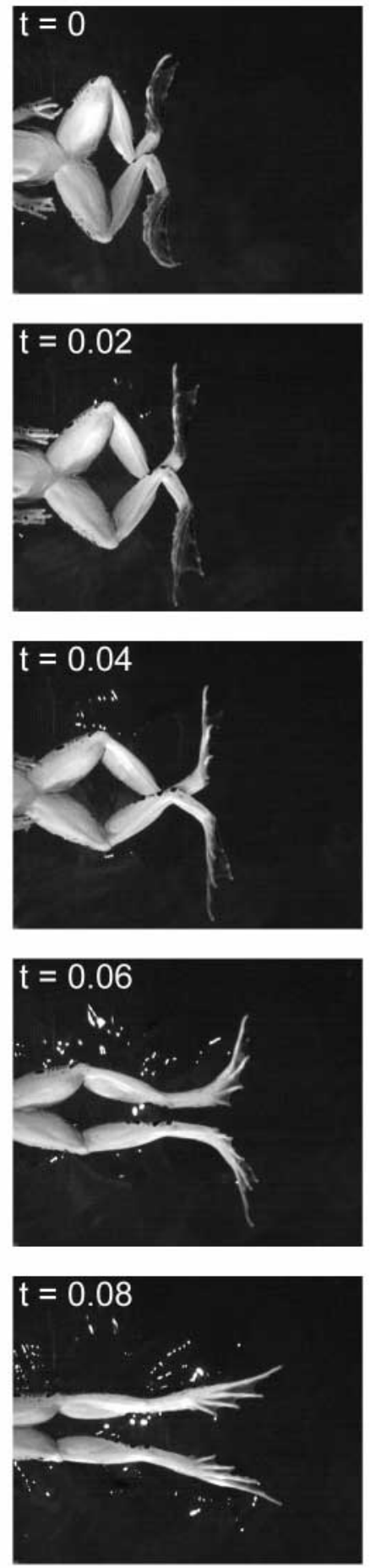
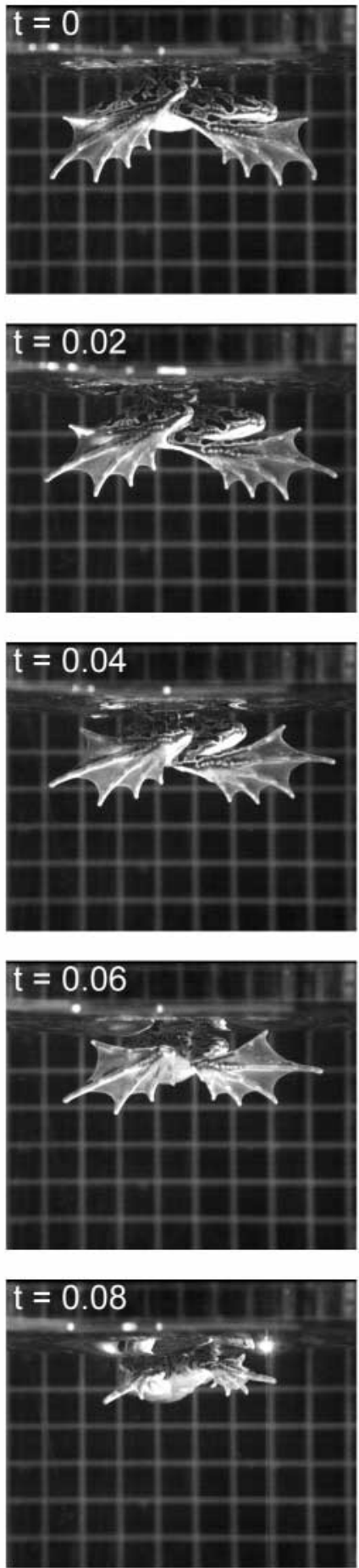
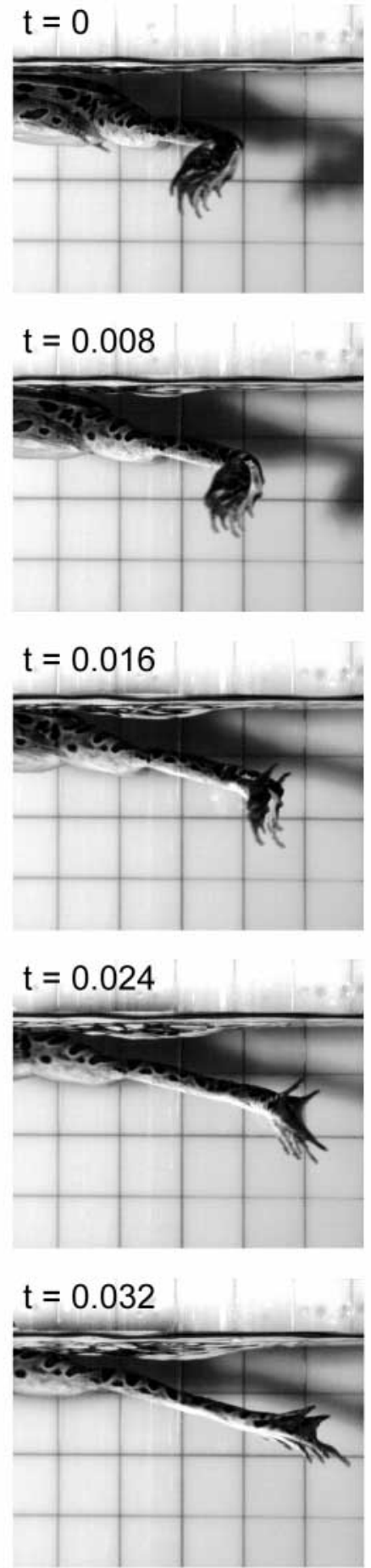

Fig. 2. Images from five stages of synchronous kicks to show basic leg and foot motion, with the ventral and caudal view from the same sequence. The lateral view is from an additional sequence, synchronized relative to the position of the legs and feet as seen from the ventral view. Note that the ankles are crossed and overlap as seen in the posterior view during the middle of the stroke, a phenomenon we saw frequently in these frogs. The feet are more or less perpendicular to the swimming direction throughout the majority of the kick. Only at the end of the kick are the feet swept medially, aligning the feet with the swimming direction. Numbers indicate the timing (s) of the image relative to the start image. 
set by the researcher. Assuming a circular vortex ring structure, with a diameter determined by the distance between the vortex centers, we calculated the momentum (circulation multiplied by the area of the ring multiplied by the density of the fluid) of the flow generated by the feet (see Drucker and Lauder, 1999). A circular vortex structure is a reasonable estimate, as the radius of the vortex ring in the vertical and horizontal views did not differ for either asynchronous or synchronous kicks [general linear model $(\mathrm{GLM}) ; P_{\mathrm{alt}}=0.471$, $\left.P_{\text {sync }}=0.939\right]$. To be able to compare our results with those in the literature, we calculated the mass-specific momentum produced during the kick. The mean force during the kick was
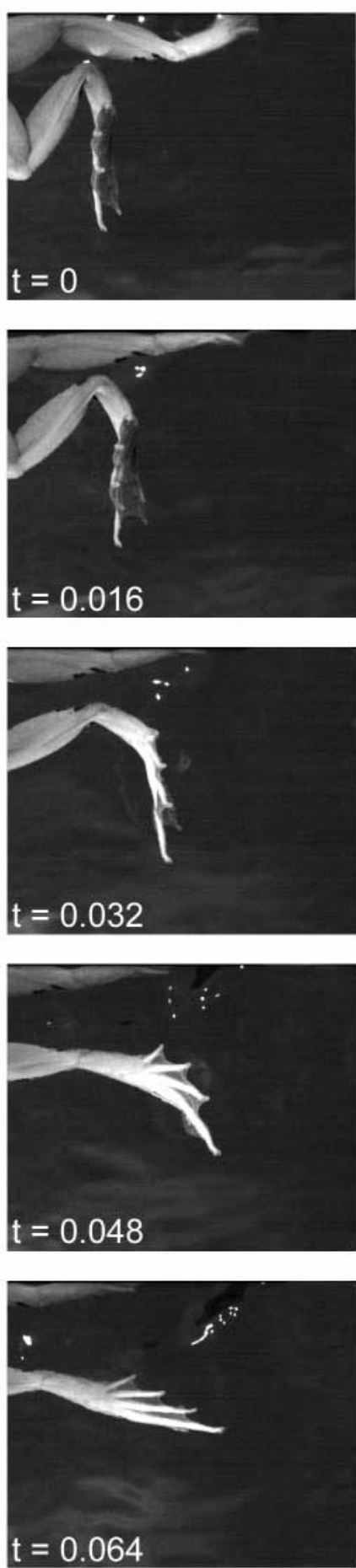
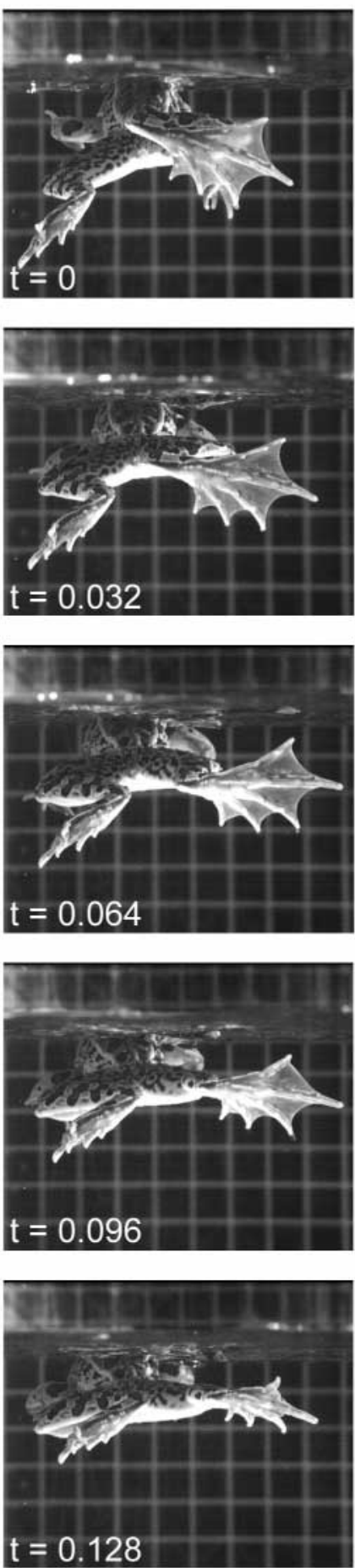
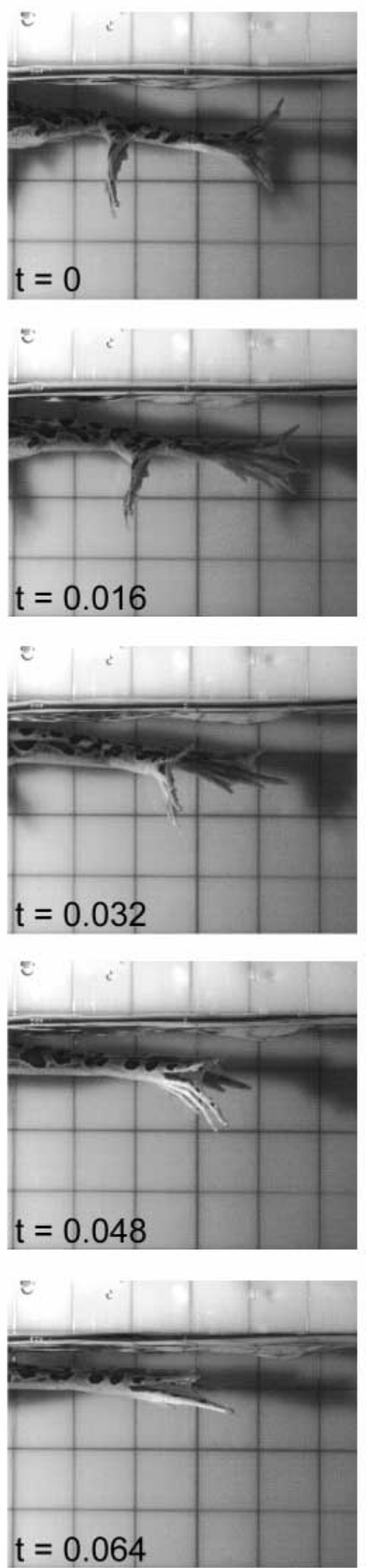

Fig. 3. Images from asynchronous kicks to show basic leg and foot motion, with the ventral and lateral view from the same sequence. The caudal view is from an additional sequence, synchronized relative to the position of the legs and feet as seen from the ventral view. Numbers indicate the timing $(s)$ of the image relative to the start image. 
calculated by dividing the momentum by the duration of the kick (e.g. Drucker and Lauder, 1999). Jet velocity was
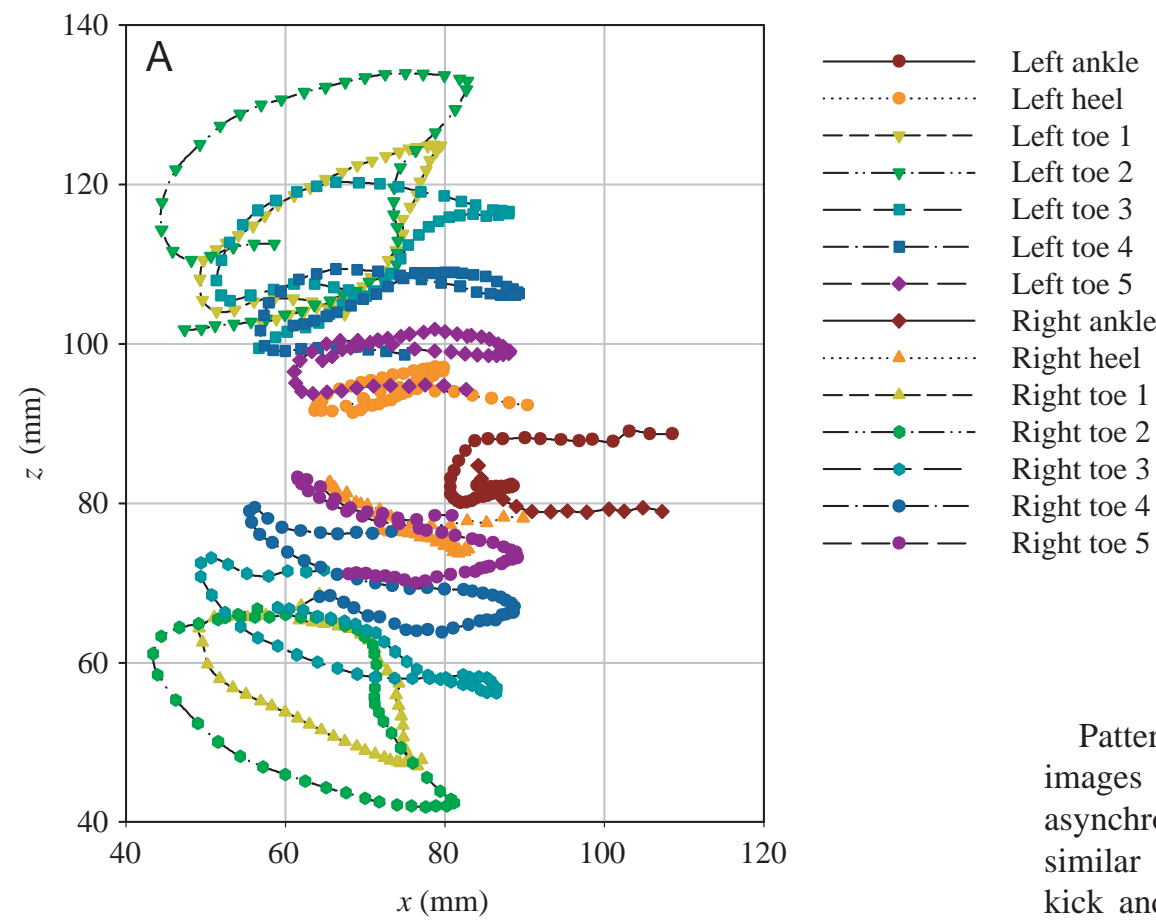

calculated as the mean velocity of the vectors in an area between the vortex centers. Jet angle relative to ring angle was calculated as the difference between the mean direction of the jet and the direction of the ring plane, determined from the position of the vortex centers. Ring angle relative to forward swimming direction was determined according to Fig. 1.

Statistical analyses were conducted in SPSS 12.0. We used GLM on $\log _{\mathrm{e}^{-}}$ transformed variables, with 'individual' as a random factor to account for individual variation. Variables were tested for equality of variance using Levene's test, and the residuals were tested for normality using the Kolmogorov-Smirnov Z-test.

\section{Results}

Patterns of limb and foot motion are presented as images for a synchronous (Fig. 2) and an asynchronous kick (Fig. 3). The motion of the feet is similar between the asynchronous and synchronous kick and the main difference is the time it takes to perform the kick (Table 1). Detailed kinematics (DLT corrected coordinates) of all toes, the heel and the ankle throughout a synchronous kick as seen from the ventral, lateral and caudal views are shown in Fig. 4. Toes are extended in preparation for the kick, and the webbing is spread as all the toes extend laterally and toes 1-3 simultaneously extend ventrally, while toe 5 moves dorsally. Throughout the majority of the kick, the feet are then held essentially perpendicular to the swimming direction. During the last part of the kick, the toes are swept medially, extending the feet posteriorly behind the outstretched legs, and the webbing collapses (toes adducted).

The velocity profile of the body and the tip of the third toe from an asynchronous and a synchronous kick are shown in Fig. 5. The average maximum swimming speed differs between gaits and, for the sequences used for DPIV, the speed is approximately 3.5 times as high during the synchronous kicks as during the asynchronous kicks. During synchronous kicks, the speed of the frog increases steeply during the first portion of the kick. However, shortly after the feet start sweeping medially, at the last part of the kick, the forward velocity starts to decrease. At this point, the momentary force produced is less than the inertial and hydrodynamical resistance of the body. During asynchronous kicks, the point at which the body starts decelerating occurs earlier during the kick, suggesting that the force produced is less relative to the resistance than during synchronous kicks. This is reasonable as
Fig. 4. Three-dimensional kinematics of the foot (relative to a stationary flow), of a representative synchronous kick as seen from the ventral (A), lateral (B) and caudal (C) views. Data points are separated by $0.004 \mathrm{~s}$. Note the relative excursions of the different toes. 
Table 1. Kinematic and hydrodynamic measurements from surface-swimming leopard frogs

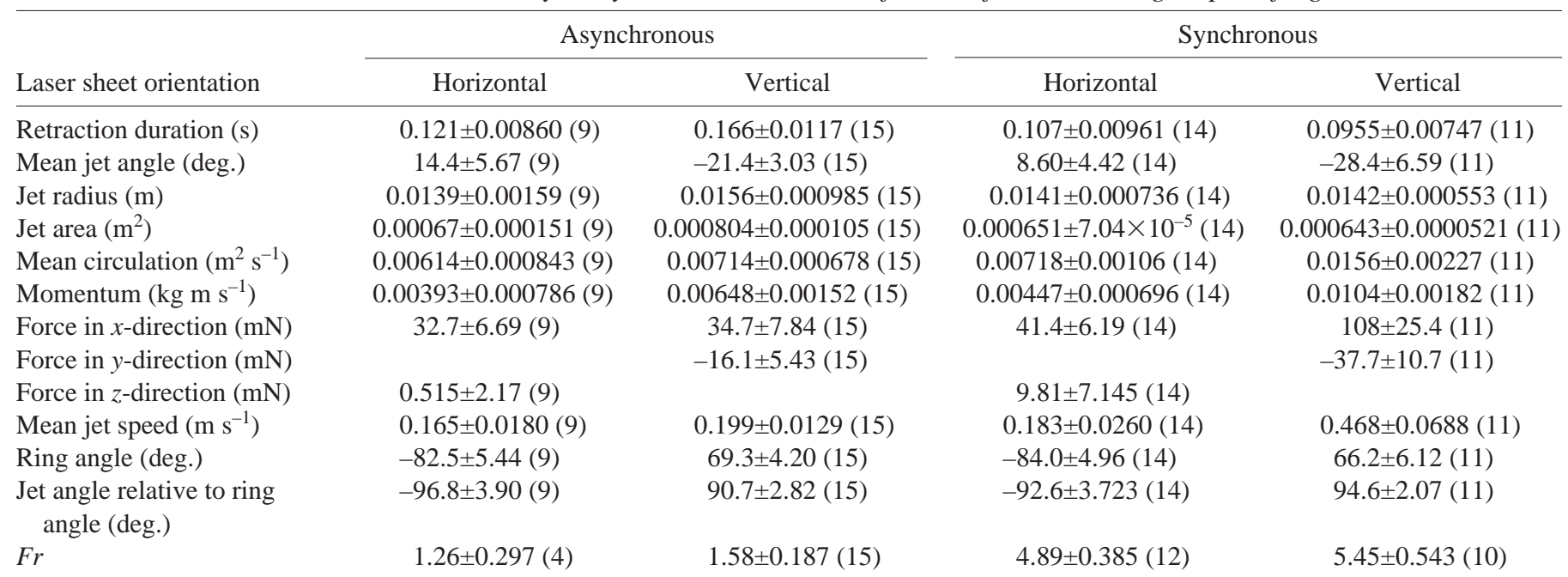

The data are presented as values per foot, except for $F r$, which is based on the velocity of the body of the frog at the end of the propulsive kick.

Values represent means \pm S.E.M. $(N)$. $F r$ is the Froude number, calculated as $F r=2 \pi U^{2} / g l$, where $U$ is the swim speed, $g$ is the gravitational acceleration and $l$ is the length of the hull (outstretched body).

during asynchronous kicks the alternate leg is in recovery phase (and hence moving forward) after the previous kick and thereby increases the resistance of the frog.

DPIV data from asynchronous kicks are presented as vector plots on top of the original video images at four different times during the kick as seen from the ventral (Fig. 6) and lateral (Fig. 7) views. In the initial phase of the kick, as seen from the ventral view, water is sucked along with the foot, and a shear layer builds up. As the kick progresses, the shear layer rolls up into an attached vortex around the edges of the feet. When the feet start sweeping medially, the distal part of this vortex is shed and, shortly thereafter, the medial part of the ring becomes visible and is shed. After the completion of the kick, each foot has produced individual vortex rings that, in the horizontal plane, are almost perpendicular to the swimming direction (Fig. 6; Table 1). In lateral view, the pattern is similar but there is a strong tendency for the lower vortex center to be shed before the upper vortex center. This results in a tilted vortex ring, with a downwarddirected jet and thus an upward-directed force acting on the frog.

DPIV data for synchronous kicks are presented as vectors on top of the calculated vorticity of the flow at three different

Fig. 5. Swimming velocity and the forward velocity of the third toe of (A) an asynchronous and (B) a powerful synchronous kick. Note the difference in magnitude of body velocity. In the synchronous kick, the foot is moving backwards relative to the water throughout nearly the entire acceleration phase of the kick, while in the asynchronous kick the body starts to decelerate when the foot is still moving backwards relative to the water. The inserted images illustrate the posture of the frog at the time indicated by the position of the waist of the frog. stages of representative kicks as seen from the ventral view (Fig. 8) and the lateral view (Fig. 9). There is also a video

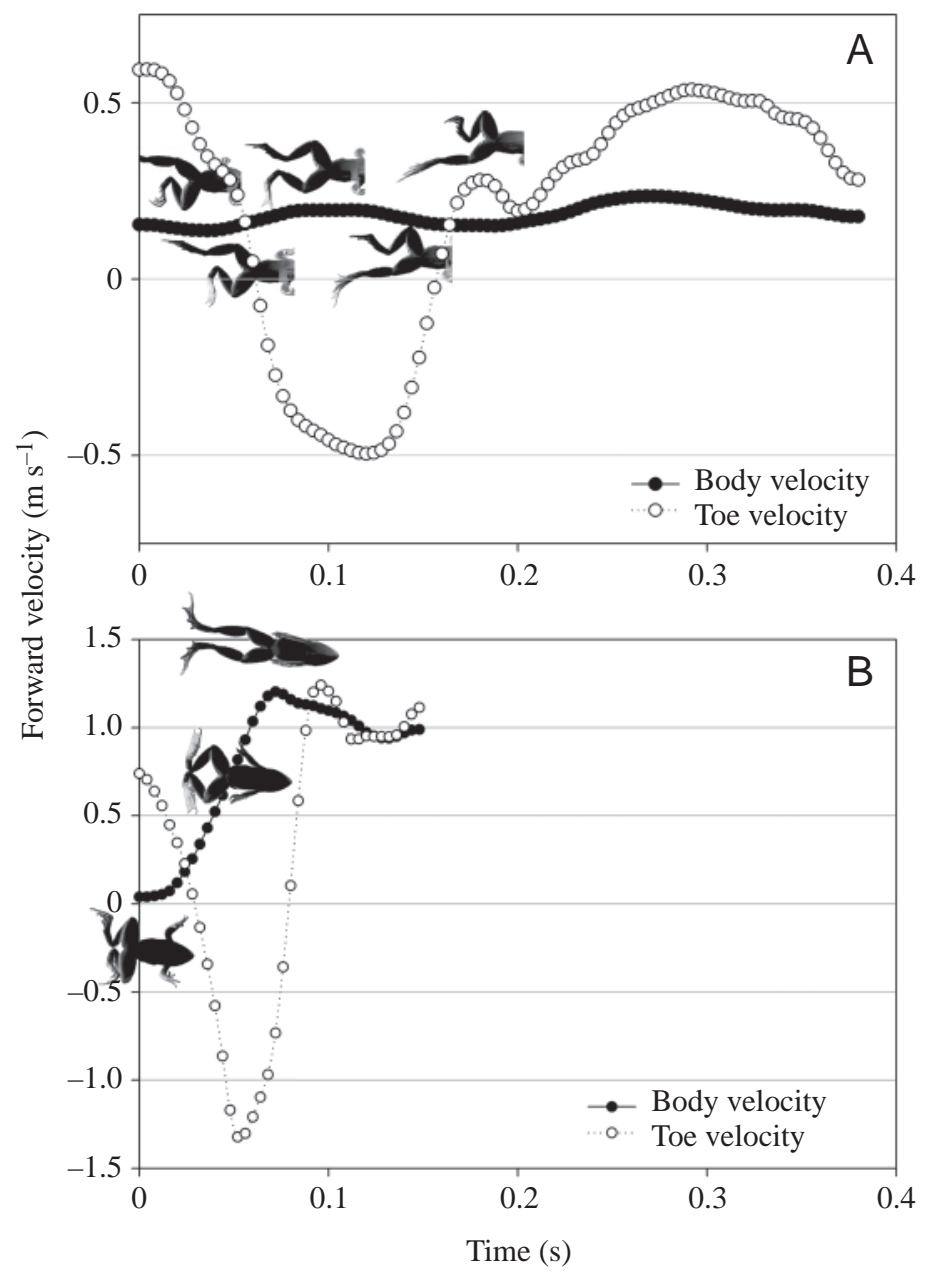


3952 L. C. Johansson and G. V. Lauder
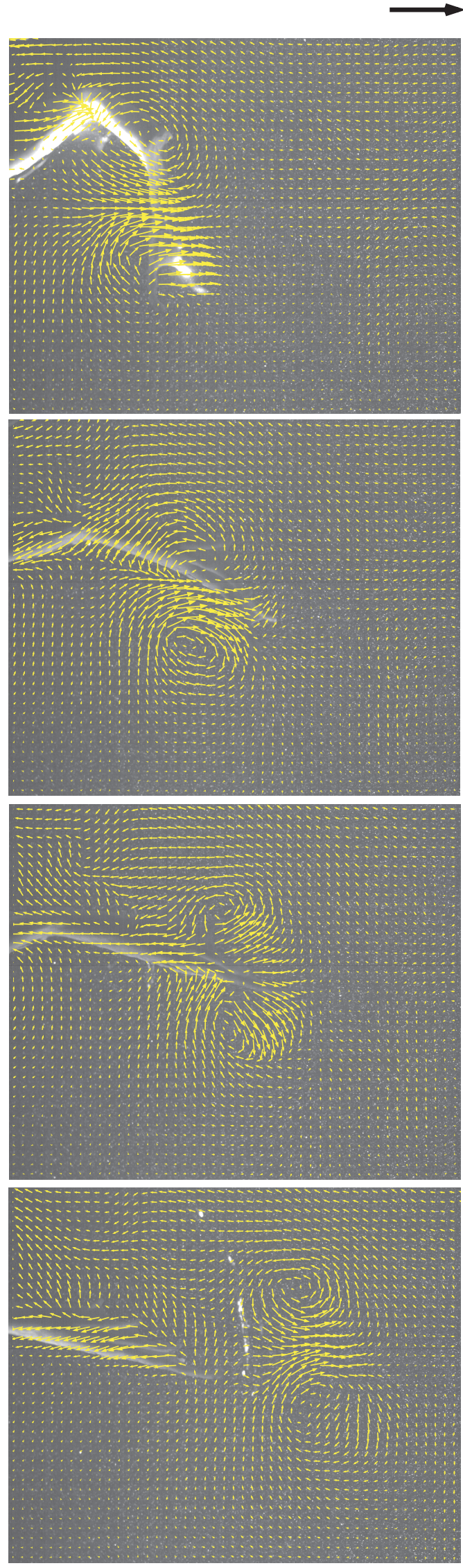

Fig. 6
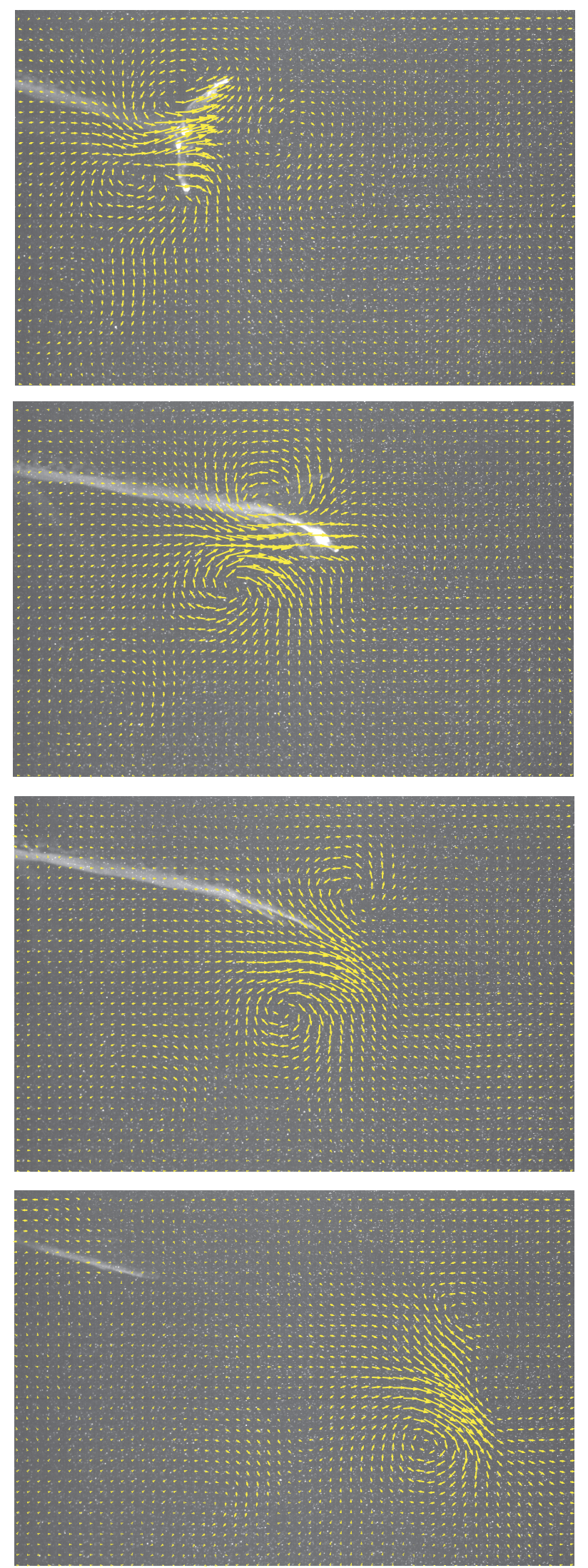

Fig. 7 
Fig. 6. Velocity vectors from an asynchronous kick based on a ventral view (horizontal light sheet; $x z$ plane) showing the buildup and shedding of the vortex ring generated by the foot. The reference vector equals $1 \mathrm{~m} \mathrm{~s}^{-1}$. The four images are separated by $0.04 \mathrm{~s}$. Velocity vectors overlying the foot represent foot movement and illustrate the velocity of the foot relative to the surrounding fluid. Free-stream flow velocity has been subtracted from each vector.

Fig. 7. Velocity vectors from an asynchronous kick based on a lateral view (vertical light sheet; $x y$ plane). The reference vector equals $1 \mathrm{~m} \mathrm{~s}^{-1}$. The four images are separated by $0.04 \mathrm{~s}$, showing the buildup and shedding of the vortex ring generated by the foot. The lower vortex center is shed briefly before the dorsal vortex center, and a discrete vortex ring with a posteroventral central jet flow of high velocity is shed into the water. Free-stream flow velocity has been subtracted from each vector.

available online as supplementary material showing the vectors superimposed on the original images of a synchronous kick, with the vectors color coded by vorticity. The buildup and shedding of the vorticity follow essentially the same patterns as for the asynchronous kick. There are two areas with counter-clockwise and clockwise vorticity linked by an area of high rearward velocity in between, indicating the generation of separate vortex rings for each foot. In the ventral view, the feet are still in the image (Fig. 8). No central rearward-directed jet between the feet is visible in the ventral view (Fig. 8). A velocity profile through the wake (Fig. 10) clearly shows two distinct jets, one for each foot; there is no single central jet. In fact, the velocity profile through the two vortex rings suggests a forward flow between the feet, not a rearward flow (Fig. 10).

Thrust (force in $x$-direction; $F_{\mathrm{x}}$ ), but not mass-specific momentum $(M)$, shows a significant positive relationship with swim speed (GLM with velocity as covariate, $P_{\mathrm{Fx}}<0.005, P_{\mathrm{M}}=0.479$; Fig. 11A,B). Furthermore, speed $(U)$ and force, but not mass-specific momentum, are significantly different between asynchronous and synchronous kicks, with higher values for synchronous kicks (GLM, $\left.P_{\mathrm{U}}<0.001, \quad P_{\mathrm{F}}<0.05, \quad P_{\mathrm{M}}=0.320\right)$. Massspecific momentum shows no correlation with the duration of the kick (GLM with stroke duration as covariate, $P_{\mathrm{M}}=0.931$; Fig. 11C).

Fig. 8. Velocity vectors from a synchronous kick based on a ventral view (horizontal light sheet; $x z$ plane) plotted on a vorticity background. The reference vector equals $1 \mathrm{~m} \mathrm{~s}^{-1}$. Red indicates counter-clockwise and blue indicates clockwise vorticity $\left(\mathrm{s}^{-1}\right)$. The three images are separated by $0.04 \mathrm{~s}$, showing the build up of the vortex ring shed by the feet. No central jet is visible between the feet, but instead each foot produces its own vortex ring with a corresponding posteriorly directed jet. The forward directed vectors (to the left of the image) and the associated vorticity are caused by the feet still being in the image. Free-stream flow velocity has been subtracted from each vector.
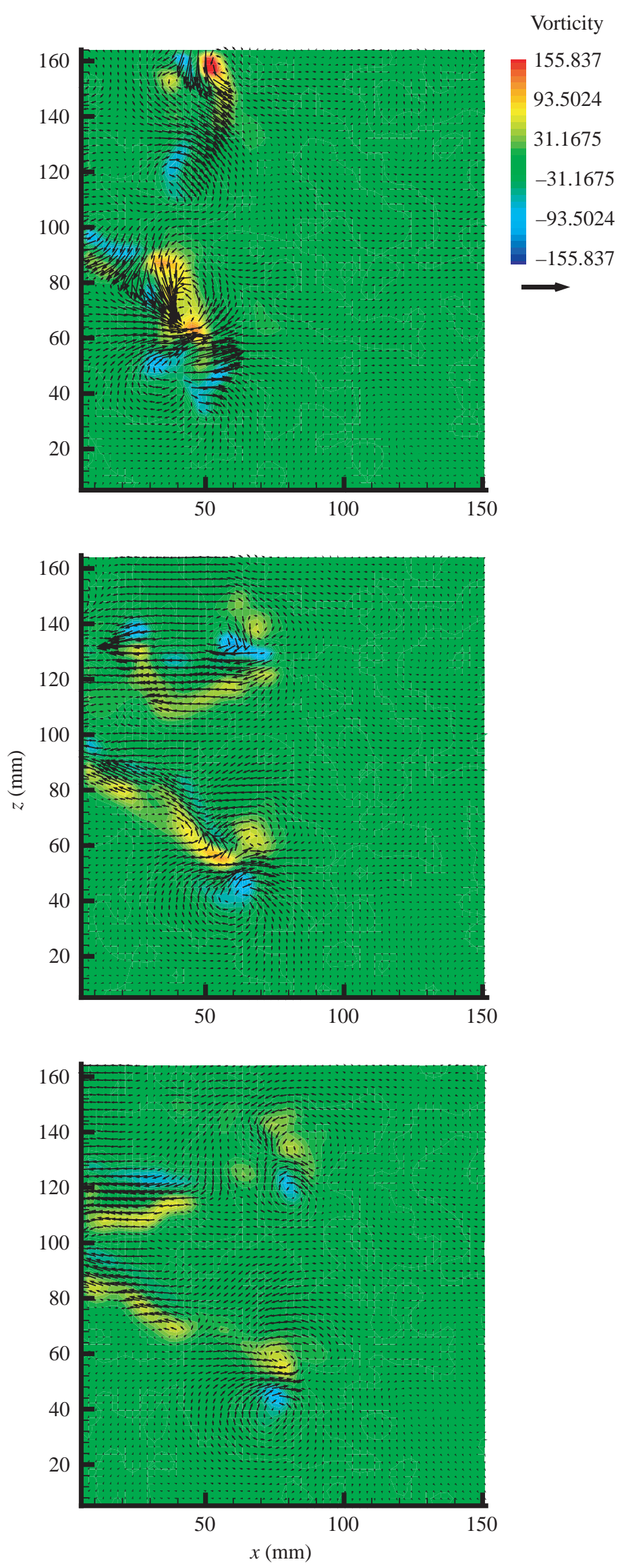


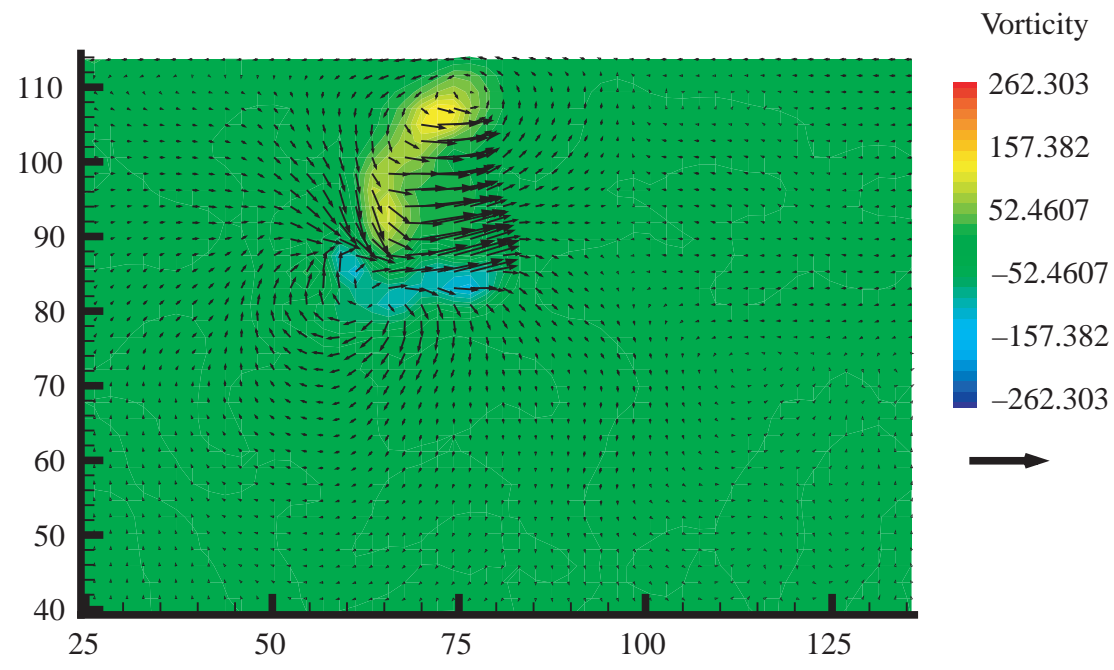

Fig. 9. Velocity vectors from a synchronous kick based on a lateral view (vertical light sheet; $x y$ plane) plotted on a vorticity background. The reference vector equals $1 \mathrm{~m} \mathrm{~s}^{-1}$. Red indicates counter-clockwise and blue indicates clockwise vorticity $\left(\mathrm{s}^{-1}\right)$. The three images are separated by $0.04 \mathrm{~s}$, showing the buildup of the vortex ring shed by the foot. In the two upper images, the foot is still in the image, affecting the estimation of the vector fields. In the top image, the vorticity centers are upstream of the foot, while in the middle image the foot is the source of the vectors pointing upstream just upstream of the vortex ring. Free-stream flow velocity has been subtracted from each vector.

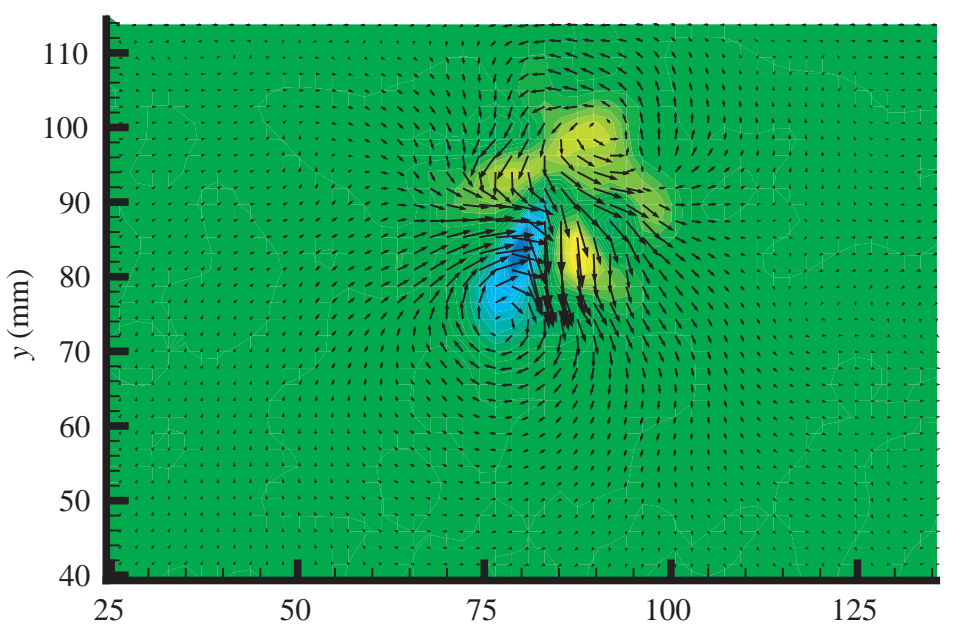

it seems as if a third hypothesis suggesting only a drag- and acceleration-reaction-based mechanism is best supported as a mechanism of force production. Also, the overall pattern of flow around the feet is very similar for the asynchronous and synchronous kicks, suggesting that the same hydrodynamic mechanism is used for both gaits. No central jet is visible in the wakes of the synchronous kicks (Fig. 9); instead, a forward flow between two distinct vortex rings is visible (Fig. 10). The lack of a central jet is logical in the sense that the velocity of the feet relative to the water is relatively low at the end of the kick and the feet are relatively far apart. Furthermore, it does not seem as if the frogs use a lift-based mechanism similar to the one suggested for birds (Johansson and Norberg, 2003), as the buildup of the vorticity is in close proximity to the feet and no shedding of a start vortex is apparent until very late in the kick. It is, however, possible that the frogs use an asynchronous shedding of the vortex ring with the vortex center at the distal tip of the foot being shed before the vortex center close to the heel. This may result in a brief period of lift production, but the purpose is more likely to facilitate the shedding of the vortex ring and reduce negative effects of acceleration reaction forces (Daniel, 1984). The flow data thus support the view that the frog kick is predominately acceleration-reaction- and drag-based, with the feet accelerating or at least moving rearwards during the entire acceleration phase of the body. According to the calculations in Daniel (1984), accelerationreaction-based thrust is maximized in the twopropulsor system when the feet are swept

\section{Discussion}

Our data do not support either of two hypotheses concerning production of a central jet between the feet (Gal and Blake, 1988a,b) or a lift-based mechanism similar to that used by some birds (Johansson and Norberg, 2003). Instead, backwards until they meet behind the animal, as is the case for the frogs. This minimizes the adverse effects of the decelerating motion of the feet at the end of the kick. However, according to the model in Daniel (1984), there should be a large lateral force on the feet at the end of the 
A

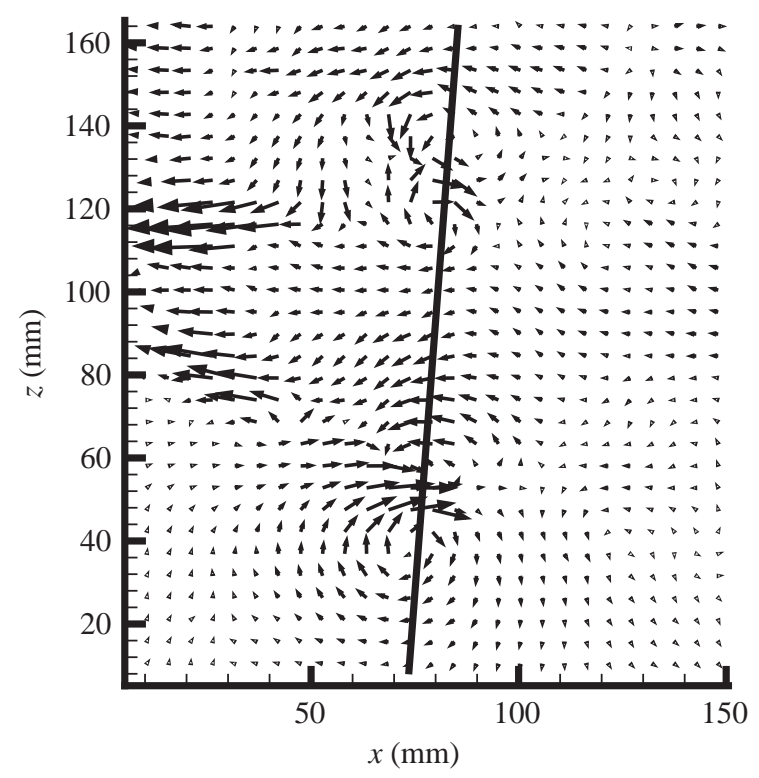

$\mathrm{B}$

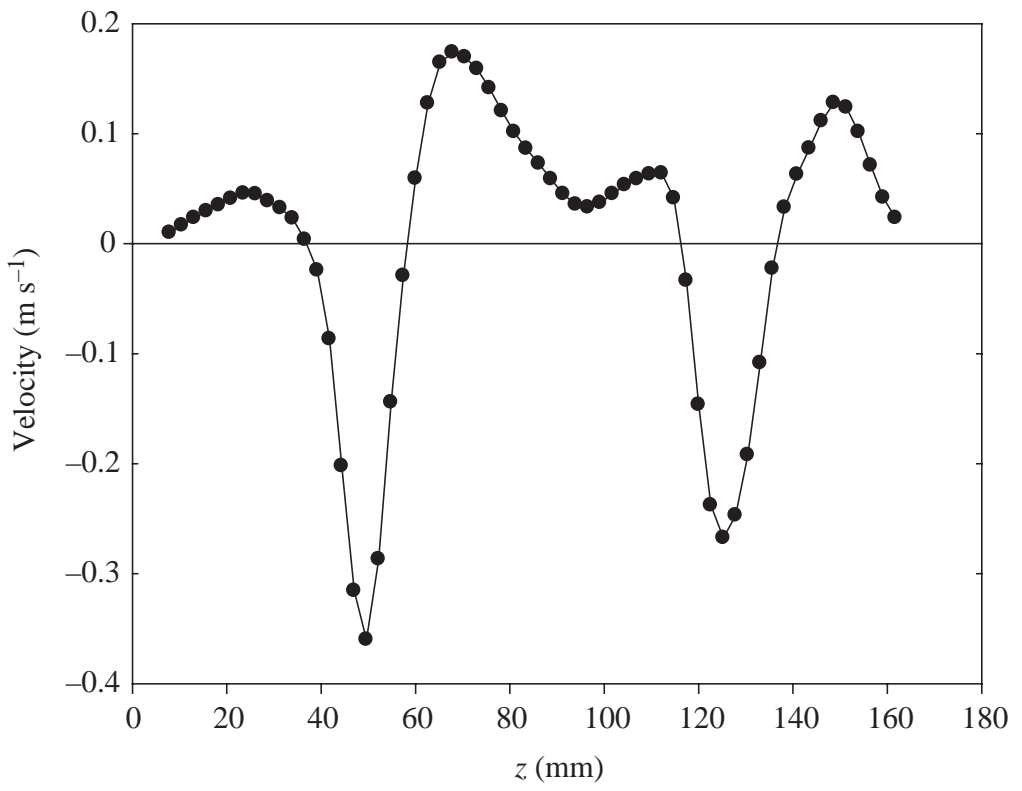

Fig. 10. Transect of the vortex wake of a synchronous kick (A) and the corresponding velocity profile perpendicular to the transect (B). Positive velocity is forward, showing an augmented forward velocity between the vortex rings. There is no backward-directed central jet produced at the end of the kick as the feet come together. If anything, there may be an interaction between the vortex rings produced during the kick, increasing the forward velocity between the vortex rings. The forward velocity between the rings may also be partly due to the drag wake of the body.

kick, which should be visible in the flow as a tilted vortex ring. This is not the case for our data (Table 1).

The frogs in our study appear to follow a different acceleration pattern for synchronous kicks than the frogs described by Gal and Blake (1988a,b). In their study, they suggest that frogs accelerate throughout the entire kick (power stroke). This could be due to interspecific differences in locomotor patterns as they studied Hymenochirus boettgeri while our study focused on Rana pipiens. However, examination of the plots of speed versus time in Gal and Blake (1988a,b) suggests that a slight deceleration is present at the end of the kick in Hymenochirus. More importantly, however, is that in Hymenochirus the velocity of the feet, at mid power stroke, was forwards relative to the still water, resulting in negative drag-based thrust ( $\mathrm{Gal}$ and Blake, 1988a,b). This is different from what is seen in our frogs, as reflected in the data shown in Fig. 5. Based on a blade element analysis of the feet of Hymenochirus, Gal and Blake (1988b) concluded that the thrust produced by drag and accelerative forces on the feet was insufficient to explain the observed acceleration pattern of the frog and suggested a squeezing jet being produced at the end of the kick. Although the velocity of the feet in our study is backwards relative to the water during the entire acceleration phase of the frog, our results suggest that a jet produced at the end of the kick may not be a reasonable explanation for the observed acceleration pattern in Hymenochirus. On the other hand, the kinematics of the fully aquatic frog Hymenochirus, presented by Gal and Blake (1988b), suggest the possibility of a more substantial use of a lift-based mechanism than do our results. In Hymenochirus there is a continuous change of the angle of the foot relative to the swimming direction during the kick and a relatively short duration of a rearward motion of the feet relative to the water (less than 50\% of the power stroke) while the feet continue to sweep medially throughout the kick, suggesting a larger dependency on lift-based forces. The foot shape in Hymenochirus is more symmetrical than the feet of R. pipiens, which are more skewed. The feet of Hymenochirus are also held in a more lateral position that makes them more symmetrical relative to the motion of the feet (L.C.J. and G.V.L., personal observations). Although this triangular shape of the feet is relatively efficient for drag-based propulsion (Blake, 1981), it also suggests the possibility of a delta wing mechanism (Johansson and Norberg, 2003). The use of a lift-based mechanism in Hymenochirus thus seems more reasonable than the formation of a jet at the end of the kick.

Gal and Blake (1988b) also suggested an interaction between the feet at the end of the kick as an alternative to the central jet hypothesis but did not further explain how such an interaction would function hydrodynamically. We do find some support for a possible interaction between the vortex rings produced by the two feet, as we observed significant positive (forward) velocities in the region between the two vortex rings (Fig. 10). This could be due to fluid dynamic interactions between the vortex rings resulting from vorticity from the ring produced by one foot enhancing the opposite sign vorticity of the ring produced by the other foot. The forward velocity between the two vortex rings is higher than the forward velocity on the outside of the two rings (Fig. 10). 

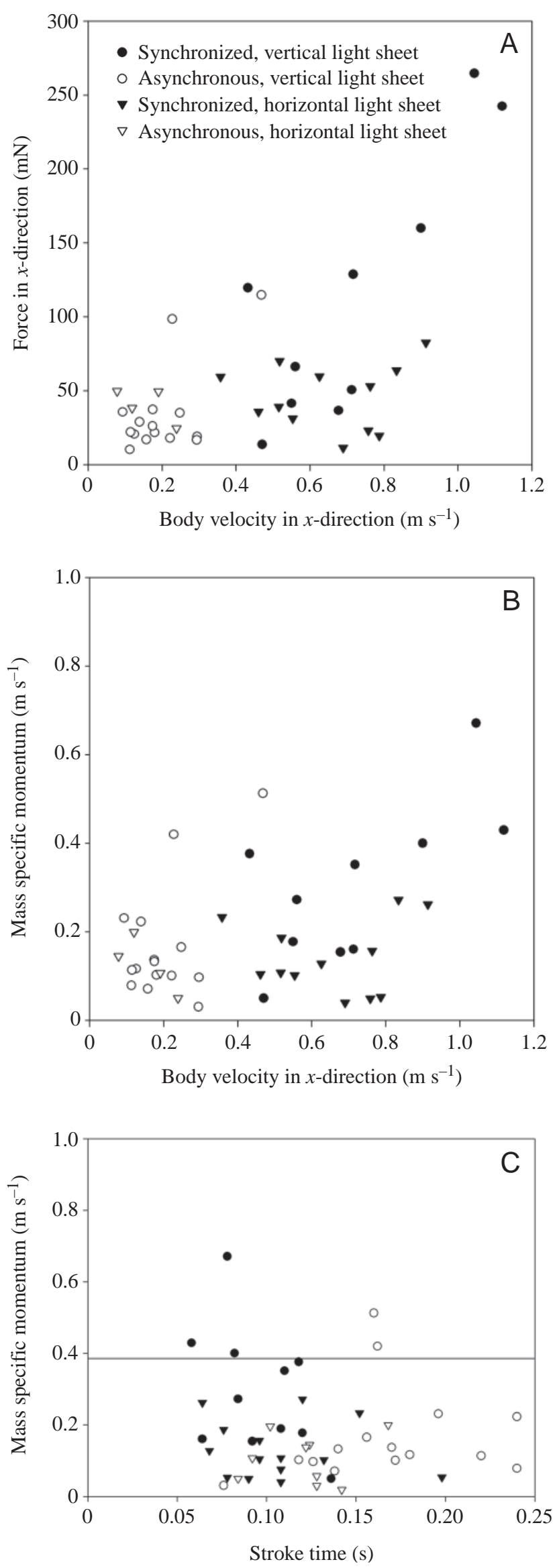

Fig. 11. (A) Forward-directed thrust per foot relative to forward swimming velocity, with higher thrust and velocity for synchronous kicks relative to asynchronous kicks. (B) Mass-specific momentum per foot relative to forward velocity and (C) duration of the power stroke, with the horizontal line in $\mathrm{C}$ representing the approximate average mass-specific momentum per foot for Rana esculenta calculated from fig. 3 in Nauwelaerts and Aerts (2003).

However, this may alternatively be an effect of the interaction between the drag wake of the frog body and the two vortex rings from the feet. Either way, this may affect the development of the rings as well as the strength of the rings and thereby the force produced. The consequence of such an interaction is not clear at this point, but we have not observed any merging of the vortex rings, and the rings produced by the feet are approximately one ring diameter apart when they are shed into the wake.

The kinematics of swimming frogs have been used to estimate forces during swimming, and a few issues regarding these kinematic data are worth noting. A different leg configuration between terrestrial and fully aquatic frog species has been noticed, with a more lateral knee position observed among aquatic frogs (Nauwelaerts and Aerts, 2003). The aquatic position is mimicked by semi-aquatic frogs when swimming (also described by Peters et al., 1996), suggesting some hydrodynamic significance. One reasonable explanation is that changing knee position reduces pitching moments,

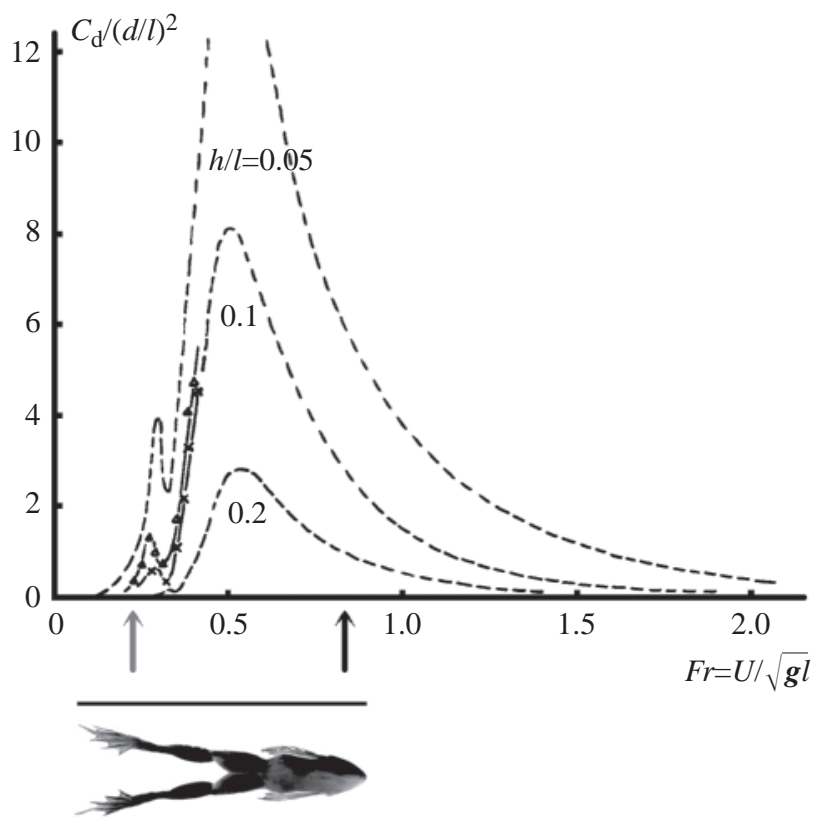

Fig. 12. The drag/wave drag $\left[C_{\mathrm{d}} /(d / l)^{2}\right]$ of a streamlined object moving at a depth $(h)$ relative to its length $(l)$ as a function of the Froude number $(F r)$. The arrows (black, synchronous kicks; gray, asynchronous kicks) indicate the average Froude number at which the frog swims during asynchronous and synchronous kicking, calculated from the velocity $(U)$ and length of the outstretched frog at the end of the kick. Graph modified from Hoerner (1965). $g$, gravitational acceleration. 
making the frog swim along a straighter path. This lateral positioning of the legs with the leg segments in approximately the same plane has prompted the suggestion that the top view alone is adequate for studies of limb kinematics (Peters et al., 1996). However, our three-dimensional kinematic analysis of the feet (Fig. 4) shows that additional views of foot and toe motion are very helpful in obtaining a more detailed understanding of what happens with the hindlimb and foot kinematics. Another kinematic feature worth noting is that the legs straighten more during swimming than during jumping (Peters et al., 1996), although the angular joint excursions are generally similar. The ankle joint is different, however, and goes through a larger angular excursion during swimming. Also, Kamel et al. (1996) showed that limb EMG patterns suggest a longer activation of the $\mathrm{M}$. plantaris longus, the muscle responsible for the straightening of the ankle joint. Together with our results, this suggests that the sweeping motion of the feet at the end of the kick is of hydrodynamic importance. At least two different reasons for this are plausible. First, bringing the feet into a streamlined position behind the body of the animal reduces drag during the glide phase. Second, the sweeping motion may facilitate the shedding of the attached vortex and reduce adverse effects of decelerating the feet at the end of the kick.

Several studies have compared swimming and jumping in frogs and found that the forces produced during swimming are less than the forces produced during jumping (Calow and Alexander, 1973; Kamel et al., 1996; Nauwelaerts and Aerts, 2003). However, these results are based on calculations of the forces from the movements of the bodies of the frogs. The data for jumping have been validated using force plates (Nauwelaerts and Aerts, 2003), and our data can be used to validate the calculations for swimming. The mass-specific momentum of synchronous kicks calculated here is slightly lower than those previously calculated from kinematics of fully submerged swimming frogs (Fig. 11C) and clearly lower than the values for jumping (Nauwelaerts and Aerts, 2003). Our result thus supports these previous comparisons of force magnitudes of aquatic and terrestrial limb motion. Also, electromyogram (EMG) data indicate more powerful kicks during jumping, with twice as strong EMG signals for jumping compared with swimming, although no correlation between EMG intensity and swim velocity or jumping distance could be found (Kamel et al., 1996). Nauwelaerts and Aerts (2003) set up a number of hypotheses to explain the difference in momentum generated during jumping and swimming. One of their hypotheses suggests large lateral forces during swimming, but our results indicate that the lateral forces are small during swimming (Table 1) as foot vortex rings generate minimal side forces (in contrast to data from fish fins in which side forces can be larger than thrust forces; Drucker and Lauder, 1999).

In agreement with previous results, we find that asynchronous kicking results in a significantly lower swimming speed than synchronous kicking (Ostry et al., 1991; Nauwelaerts and Aerts, 2002). Here, we propose, based on the physical properties of water and the resistance to body movement close to the surface, that the non-overlapping swim speeds between the two gaits may, in surface swimming, be a consequence of wave resistance. When swimming at the surface, drag is increased substantially due to the formation of waves. The wave resistance is a function of the Froude number, which is a measure of the speed $(U)$ relative to the gravity $(g)$ and the length $(l)$ of an object (Hoerner, 1965). In our study, the frogs swam below hull speed (the speed where the wave length of the bow wave is the same as the length of the hull, which is often seen as the 'maximum' speed of a displacement hull) when using asynchronous kicks. Swimming speed during synchronous kicks was substantially higher than the hull speed, calculated from the outstretched length of the frog at the end of the kick. Furthermore, the Froude number calculated according to Hoerner (1965) $\left[\mathrm{Fr}=\mathrm{U} /(\boldsymbol{g l})^{0.5}\right]$ suggests that the frogs swim at such a high speed that waves do not have time to form, resulting in a lower wave drag than at speeds close to hull speed (Fig. 12). Thus, the fast acceleration and high speed resulting from the synchronous kick enable frogs to make a faster escape than would have otherwise been the case.

Although we have used traditional, steady-state, fluiddynamic terminology to illustrate different hypotheses for the force generation, the force generation in swimming frogs does not seem to be fully explainable in these terms. Instead, the force is, as in many other biological systems, generated by attached vortices and/or vorticity centers in close proximity to the propulsive appendage (e.g. Ellington et al., 1996; Birch and Dickinson, 2001; Usherwood and Ellington, 2002). The exact consequence of limb kinematics on the generation and efficiency of the vortex-based propulsive system in frogs is still unclear and needs to be tested using mechanical or computational models that permit relatively easy manipulation of kinematic parameters. This is especially true for systems such as frog limbs that involve strong acceleration and deceleration within the time period of the force generation.

We are grateful to all the people in the Lauder lab, and especially E. Tytell, for invaluable help with PIV data processing and Matlab programming, and T. Hsieh for help with Matlab and animal care. This study was funded by NSF IBN0316675 to G.V.L. and by Lars Hiertas Minne to L.C.J. and was part of a Post-doc of L.C.J., funded by the Wenner-Gren foundations.

\section{References}

Agui, J. C. and Jimenez, J. (1987). On the performance of particle tracking. J. Fluid Mech. 185, 447-468.

Birch, J. M. and Dickinson, M. H. (2001). Spanwise flow and the attachment of the leading-edge vortex on insect wings. Nature 412, 729-733.

Blake, R. W. (1981). Influence of pectoral fin shape on thrust and drag in labriform locomotion. J. Zool. Lond. 194, 53-66.

Calow, L. J. and Alexander, R. McN. (1973). A mechanical analysis of a hind leg of a frog (Rana temporaria). J. Zool. Lond. 171, 293-321. 


\section{L. C. Johansson and G. V. Lauder}

Daniel, T. L. (1984). Unsteady aspects of aquatic locomotion. Am. Zool. 24, 121-134.

Drucker, E. G. and Lauder, G. V. (1999). Locomotor forces on a swimming fish: three-dimensional vortex wake dynamics quantified using digital particle image velocimetry. J. Exp. Biol. 202, 2393-2412.

Drucker, E. G. and Lauder, G. V. (2002). Experimental hydrodynamics of fish locomotion: functional insights from wake visualization. Int. Comp. Biol. 42, 243-257.

Ellington, C. P., van den Berg, C., Willmott, A. P. and Thomas, A. L. R. (1996). Leading-edge vortices in insect flight. Nature 384, 626-630.

Emerson, S. B. and De Jongh, H. J. (1980). Muscle activity at the ilio-sacral articulation of frogs. J. Morphol. 166, 129-144.

Gal, J. M. and Blake, R. W. (1988a). Biomechanics of frog swimming. I. Estimation of the force generated by Hymenochirus boettgeri. J. Exp. Biol. 138, 399-411.

Gal, J. M. and Blake, R. W. (1988b). Biomechanics of frog swimming. II. Mechanics of the limb-beat cycle in Hymenochirus boettgeri. J. Exp. Biol. 138, 413-429.

Gillis, G. B. and Biewener, A. A. (2000). Hindlimb extensor muscle function during jumping and swimming in the toad (Bufo marinus). J. Exp. Biol. 203, 3547-3563.

Gillis, G. B. and Blob, R. W. (2001). How muscles accommodate movement in different physical environments: aquatic vs. terrestrial locomotion in vertebrates. Comp. Biochem. Physiol. A 131, 61-75.

Hart, D. P. (2000). Super-resolution PIV by recursive local-correlation. J. Visual. 3, 187-194.

Hoerner, S. F. (1965). Fluid-Dynamic Drag. Bakersfield, CA: Hoerner Fluid Dynamics.

Johansson, L. C. and Norberg, R. Å. (2003). Delta-wing function of webbed feet gives hydrodynamic lift for swimming propulsion in birds. Nature $\mathbf{4 2 4}$, 65-68.

Kamel, L. T., Peters, S. E. and Bashor, D. P. (1996). Hopping and swimming in the Leopard frog, Rana pipiens: II. A comparison of muscle activities. J. Morphol. 230, 17-31.

Kargo, W. J., Nelson, F. and Rome, L. C. (2002a). Jumping in frogs: assessing the design of the skeletal system by anatomically realistic modeling and forward dynamic simulation. J. Exp. Biol. 205, 1683-1702.
Kargo, W. J. and Rome, L. C. (2002b). Functional morphology of proximal hindlimb muscles in the frog Rana pipiens. J. Exp. Biol. 205, 1987-2004.

Lauder, G. V. (2000). Function of the caudal fin during locomotion in fishes: kinematics, flow visualization, and evolutionary patterns. Am. Zool. 40, 101122.

Lauder, G. V. and Drucker, E. (2002). Forces, fishes, and fluids: hydrodynamic mechanisms of aquatic locomotion. News Physiol. Sci. 17, 235-240.

Lauder, G. V., Nauen, J. and Drucker, E. G. (2002). Experimental hydrodynamics and evolution: function of median fins in ray-finned fishes. Int. Comp. Biol. 42, 1009-1017.

Lutz, G. J. and Rome, L. C. (1994). Built for jumping: the design of the frog muscular system. Science 263, 370-372.

Marsh, R. L. (1994). Jumping ability of anuran amphibians. Adv. Vet. Sci. Comp. Med. 38B, 51-111.

Marsh, R. L. and John-Alder, H. B. (1994). Jumping performance of hylid frogs measured with high-speed cine film. J. Exp. Biol. 188, 131-141.

Nauen, J. C. and Lauder, G. V. (2002). Hydrodynamics of caudal fin locomotion by chub mackerel, Scomber japonicus (Scombridae). J. Exp. Biol. 205, 1709-1724.

Nauwelaerts, S. and Aerts, P. (2002). Two distinct gait types in swimming frogs. J. Zool. Lond. 258, 183-188.

Nauwelaerts, S. and Aerts, P. (2003). Propulsive impulse as a covarying performance measure in the comparison of the kinematics of swimming and jumping in frogs. J. Exp. Biol. 206, 4341-4351.

Nauwelaerts, S., Aerts, P. and D'Août, K. (2001). Speed modulation in swimming frogs. J. Motor Behav. 33, 265-272.

Ostry, D. J., Feldman, A. G. and Flanagan, J. R. (1991). Kinematics and control of frog hindlimb movements. J. Neurophys. 65, 547-562.

Peters, S. E., Kamel, L. T. and Bashor, D. P. (1996). Hopping and swimming in the Leopard frog, Rana pipiens: I. Step cycles and kinematics. J. Morphol. 230, 1-16.

Tytell, E. D. and Lauder, G. V. (2004). Hydrodynamics of eel swimming. I. Wake structure. J. Exp. Biol. 207, 1825-1841.

Usherwood, J. R. and Ellington, C. P. (2002). The aerodynamics of revolving wings. II. Propeller force coefficients from mayfly to quail. J. Exp. Biol. 205, 1565-1576. 